MATHEMATICS OF COMPUTATION

Volume 77, Number 264, October 2008, Pages 2155-2171

S $0025-5718(08) 02107-8$

Article electronically published on March 19, 2008

\title{
A STABLE TEST FOR STRICT SIGN REGULARITY
}

\author{
V. CORTÉS AND J. M. PEÑA
}

\begin{abstract}
A stable test to check if a given matrix is strictly sign regular is provided. Among other nice properties, we prove that it has an optimal growth factor. The test is compared with other alternative tests appearing in the literature, and its advantages are shown.
\end{abstract}

\section{InTRODUCTION AND MOTIVATIONS}

An $m \times n$ matrix $A$ is strictly sign regular (SSR) if, for each $k(1 \leq k \leq$ $\min \{m, n\})$, all $k \times k$ submatrices of $A$ have a determinant with the same strict sign (see [13). The interest in these matrices comes from their characterizations as variation-diminishing linear maps: the number of sign changes in the consecutive components of the image of a vector is bounded above by the number of sign changes in the consecutive components of the vector (cf. Theorem 5.6 of [1]). The theory of variation-diminishing transformations was originated by Schoenberg [16]. Many applications of these transformations can be found in 9] and [1. Let us now mention some examples showing the usefulness of providing efficient tests to check if a given matrix is SSR. These examples belong to different branches of mathematics and, more generally, to scientific computing, and they motivate the interest of the test proposed in this paper:

- In computer aided geometric design, it is convenient that the curve or surface imitate the shape of the corresponding control polygon or control net. In order to assure this property one has to check that the basis provides a shape preserving representation, which reduces in turn to check that certain associated matrices are SSR. See 12 and the references therein for more details.

- In statistics, we can assure basic properties of the hypothesis test provided by a family of densities such that their associated collocation matrices are SSR (see 2]).

- In approximation theory, Descartes' systems (which satisfy Descartes' rule of signs) are characterized by the strict sign regularity of its collocation matrices. Moreover, the existence of these systems in a space of functions is closely related with the existence of interpolatory systems (see [3]).

Received by the editor November 17, 2007.

2000 Mathematics Subject Classification. Primary 65F05, 65F40, 15A48.

Key words and phrases. Strictly sign regular matrices, Neville elimination, pivoting strategies.

This research has been partially supported by the Spanish Research Grant MTM2006-03388 and by Gobierno de Aragón and Fondo Social Europeo. 
A very important subclass of the strictly sign regular matrices is formed by the totally positive matrices. A matrix is totally positive if all its minors are positive. In the literature these matrices have also been called strictly totally positive matrices. On the other hand, a matrix is totally negative if all its minors are negative. In [7] one can find a characterization of totally negative matrices, which were called strictly totally negative. In [5], several aspects of totally negative matrices were studied.

A test of $\mathcal{O}\left(m^{5}\right)$ arithmetic floating point operations to check if an $m \times n$ matrix $(m \geq n)$ is totally positive can be derived from Theorem 1 of [11] through Gauss elimination. Example 4.3 shows that Gauss elimination or Gauss elimination with partial pivoting are not adequate to derive an economic test to check strict sign regularity, in contrast to Neville elimination. Roughly speaking, Neville elimination is an elimination procedure which produces zeros in each column by subtracting from each row an adequate multiple of the previous one, whereas Gauss elimination produces zeros in each column by substracting from each row an appropriated multiple of a fixed row, called a pivot row. See [6] for more details and about advantages of Neville elimination when dealing with totally positive matrices. A test of $\mathcal{O}\left(n^{4}\right)$ arithmetic floating point operations to check if a square $n \times n$ matrix is SSR was proposed in [7. Here we propose an alternative test with several advantages over the test of [7]. Our new test uses Neville elimination, but it also uses a pivoting strategy, recently introduced in [4 and called two-determinant pivoting. In Theorem 6.1 we provide a new advantage of this strategy when dealing with SSR matrices: it has optimal growth factor. Let us recall that the growth factor of a numerical algorithm is the quotient between the $\infty$-norm of the output and the $\infty$-norm of the initial data. In general, a small growth factor avoids overflow and is an indicator of stability. Other recent tests controlling the growth factor have been provided for the nonsingular $M$-matrices in [15] and for checking the Routh-Hurwitz conditions in [14.

We show in Theorem 3.2 that SSR matrices can be characterized through Neville elimination with two-determinant pivoting. This fact is used in the test proposed in Section 4 to check if a given $m \times n$ matrix is SSR. The new test reduces the computational cost of the test of [7. A detailed analysis of the computational cost of the new test is performed in Section 5. As commented above, the new test has optimal growth factor. In contrast, in Example 6.2 we show that the test used in [7] can have a growth factor arbitrarily large even for $2 \times 2$ matrices. In addition, we also prove in Section 6 that, for floating point arithmetic of sufficiently high precision, applying the new test to an SSR matrix will always provide an affirmative answer. We finish Section 6 with an example illustrating with an SSR matrix (constructed in the most simple way) the higher accuracy of our test over the test provided in [7.

In scientific computing new interest on totally positive matrices and some other SSR matrices (see [10]) comes from the fact that Neville elimination (with certain pivoting strategies if the matrix is not totally positive) can provide factorizations in terms of bidiagonal matrices (and permutation matrices) which, in turn, permit accurate computations. The matrix factorization associated with our test can also be used for this purpose. This application will be analyzed by the authors in a future work. 


\section{NeVille Elimination AND TWO-DETERMinANT PIVOTING FOR SSR MATRICES}

Given $k, m \in N, k \leq m, Q_{k, m}$ will denote the set of strictly increasing sequences of $k$ natural numbers less than or equal to $m$. For each $\alpha=\left(\alpha_{1}, \ldots, \alpha_{k}\right) \in Q_{k, m}$, its dispersion number $d(\alpha)$ is defined by

$$
d(\alpha):=\sum_{i=1}^{k-1}\left(\alpha_{i+1}-\alpha_{i}-1\right)=\alpha_{k}-\alpha_{1}-(k-1),
$$

with the convention $d(\alpha)=0$ for $\alpha \in Q_{k, m}$. Let us observe that $d(\alpha)=0$ means that $\alpha$ consists of $k$ consecutive integers. Let $m, n, k, l$ be natural numbers with $k \leq m$ and $l \leq n$, let $A$ be a real $m \times n$ matrix, and let $\alpha \in Q_{k, m}$ and $\beta \in Q_{l, n}$. Then $A[\alpha \mid \beta]$ is by definition the $k \times l$ submatrix of $A$ containing rows numbered by $\alpha$ and columns numbered by $\beta$. When $\alpha=\beta, A[\alpha \mid \alpha]$ is simply denoted by $A[\alpha]$.

Neville elimination is a procedure to create zeros in a matrix by means of adding to a given row a suitable multiple of the previous one. Given an $m \times n$ matrix $A=$

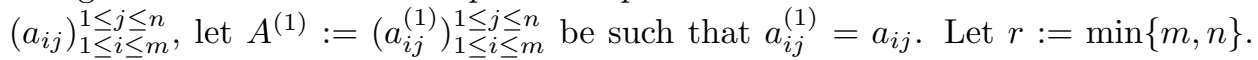
Under the assumptions of this paper, the Neville elimination of $A$ with a pivoting strategy produces a sequence of matrices as follows:

$$
A=A^{(1)} \longrightarrow \tilde{A}^{(1)} \longrightarrow A^{(2)} \longrightarrow \tilde{A}^{(2)} \longrightarrow \cdots \longrightarrow A^{(r)}=\tilde{A}^{(r)},
$$

such that $A^{(t)}=\left(a_{i j}^{(t)}\right)_{1 \leq i \leq m}^{\substack{1 \leq j \leq n \\ 1 \leq i \leq m}}$ has zeros below its main diagonal in the first $t-1$ columns and $A^{(t)}[t, \ldots, m \mid t, \ldots, n]$ only has nonzero entries. The matrix $\tilde{A}^{(t)}=$ $\left(\tilde{a}_{i j}^{(t)}\right)_{1 \leq i \leq m}^{1 \leq j \leq n}$ is obtained from the matrix $A^{(t)}$ by reordering the rows $t, t+1, \ldots, m$ of $A^{(t)}$ according to the given pivoting strategy. The matrix $A^{(t+1)}$ is obtained from $\tilde{A}^{(t)}(1 \leq t \leq r-1)$ according to the formula

$$
a_{i j}^{(t+1)}:= \begin{cases}\tilde{a}_{i j}^{(t)}, & i=1,2, \ldots, t, \\ \tilde{a}_{i j}^{(t)}-\frac{\tilde{a}_{i t}^{(t)}}{\tilde{a}_{i-1, t}^{(t)}} \tilde{a}_{i-1, j}^{(t)}, & t+1 \leq i \leq m, \quad t+1 \leq j \leq n, \\ 0, & t+1 \leq i \leq m, \quad 1 \leq j \leq t .\end{cases}
$$

In this process the element

$$
p_{i j}:=\tilde{a}_{i j}^{(j)}, \quad 1 \leq j \leq n, \quad j \leq i \leq m,
$$

will be called the $(i, j)$ pivot of the Neville elimination of $A$. Observe that the computational cost of Neville elimination coincides with that of Gauss elimination.

By a signature sequence we mean an (infinite) real sequence $\varepsilon=\left(\varepsilon_{i}\right)$ with $\left|\varepsilon_{i}\right|=$ $1, i=1,2, \ldots$ An $m \times n$ matrix $A$ verifying $\varepsilon_{k} \operatorname{det} A[\alpha \mid \beta]>0$ for all $\alpha \in Q_{k, m}, \beta \in$ $Q_{k, n}$ and for $k=1, \ldots, r$ is called strictly sign regular with signature $\varepsilon$ and will be denoted by SSR. If we write $\varepsilon_{k}(\mathrm{~A})=+1$ (resp., $\varepsilon_{k}(\mathrm{~A})=-1$ ) we mean that all $k \times k$ submatrices of $A$ have positive (resp., negative) determinants.

Let us recall that for $\gamma \in Q_{k, n}(k \leq n)$ and an $n \times n$ matrix $C$ with $C[\gamma]$ invertible, the Schur complement of $C[\gamma]$ in $C$, denoted by $C / C[\gamma]$, is defined as

$$
C / C[\gamma]=C\left[\gamma^{\prime}\right]-C\left[\gamma^{\prime} \mid \gamma\right](C[\gamma])^{-1} C\left[\gamma \mid \gamma^{\prime}\right]
$$

Then

$$
\operatorname{det}(C / C[\gamma])=\frac{\operatorname{det} C}{\operatorname{det} C[\gamma]}
$$


We denote by $P_{t}:=\left(\delta_{m-t+2-i, j}\right)_{1 \leq i, j \leq m-t+1}$ the reverse identity matrix $(m-$ $t+1) \times(m-t+1)$. Let $A$ be an $m \times n$ matrix and $1 \leq t \leq r-1$ with $r=\min \{m, n\}$. When all the entries of $A^{(t)}[t, \ldots, m \mid t, \ldots, n]$ are nonzero, then we can apply the criterion of the two-determinant pivoting strategy to obtain $\tilde{A}^{(t)}[t, \ldots, m \mid t, \ldots, n]$ from a reordering of the rows of $A^{(t)}[t, \ldots, m \mid t, \ldots, n]$ as follows:

If $t \neq r$, then we compute the determinant

$$
d_{1}:=\operatorname{det}\left(\begin{array}{cc}
a_{t t}^{(t)} & a_{t, t+1}^{(t)} \\
a_{t+1, t}^{(t)} & a_{t+1, t+1}^{(t)}
\end{array}\right) .
$$

- If $d_{1}>0$, then we do not perform row exchanges, that is, $\tilde{A}^{(t)}:=A^{(t)}$.

- If $d_{1}<0$, then we reverse the ordering of the rows from $t$ to $m$, that is, $\tilde{A}^{(t)}[t, \ldots, m \mid t, \ldots, n]:=P_{t} \cdot A^{(t)}[t, \ldots, m \mid t, \ldots, n]$.

If $t=r$, then $\tilde{A}^{(t)}:=A^{(t)}$.

The following result shows that strict sign regularity is inherited by all matrices $A^{(t)}[t, \ldots, m \mid t, \ldots, n]$ when we apply Neville elimination with two-determinant pivoting, and so the pivoting strategy is well defined for SSR matrices. Observe that two-determinant pivoting increases the cost of Neville elimination of an $m \times n$ matrix at most $r-1$ substractions and $2 r-2$ multiplications where $r:=\min \{m, n\}$.

Proposition 2.1. Let $A$ be an $m \times n$ SSR matrix with signature $\varepsilon$ and let us apply Neville elimination with two-determinant pivoting. Let $r:=\min \{m, n\}$. Then, for all $t \in\{1, \ldots, r\}$, all the matrices $A^{(t)}[t, \ldots, m \mid t, \ldots, n]$ are $S S R$ and $\varepsilon_{1}\left(A^{(t)}\right)=$ $\varepsilon_{1}(A)$.

Proof. Let us prove the result by induction on $r$. The result is trivial for $r=1$. Let us assume that it holds for $r-1$, and let us prove it for $r$.

Since $A$ is SSR, when we apply Neville elimination with two-determinant pivoting, the resulting matrix $\tilde{A}^{(1)}$ is SSR with $\varepsilon_{2}\left(\tilde{A}^{(1)}\right)=+1$. Let us consider $\alpha_{1}, \beta_{1}$ with $2 \leq \alpha_{1} \leq m$ and $2 \leq \beta_{1} \leq n$. Since $\varepsilon_{2}\left(\tilde{A}^{(1)}\right)=+1$, applying formula (2.4) (taking $C:=\tilde{A}^{(1)}\left[\alpha_{1}-1, \alpha_{1} \mid 1, \beta_{1}\right]$ and $\gamma:=(1)$ ), we can deduce that all the elements of the matrix $A^{(2)}[2, \ldots, m \mid 2, \ldots, n]$ have the same strict sign which is $\varepsilon_{1}\left(\tilde{A}^{(1)}\right)\left(=\varepsilon_{1}(A)\right)$.

Now, we consider $\alpha \in Q_{s, m}, \beta \in Q_{s, n}$ with $1 \leq s \leq r-1,2 \leq \alpha_{1} \leq m-s+1$, $2 \leq \beta_{1} \leq n-s+1$ and $d(\alpha)=d(\beta)=0$. Using (2.4) (taking $C:=\tilde{A}^{(1)}\left[\alpha_{1}-1, \alpha \mid 1, \beta\right]$ and $\gamma:=(1)$ ), we can observe that all the minors $\operatorname{det} A^{(2)}[\alpha \mid \beta]$ of order $s$ with consecutive rows and columns of the matrix $A^{(2)}[2, \ldots, m \mid 2, \ldots, n]$ have the same strict sign which is $\varepsilon_{s+1}\left(\tilde{A}^{(1)}\right) / \varepsilon_{1}\left(\tilde{A}^{(1)}\right)$. By Theorem 2.5 of [1], we conclude that $A^{(2)}[2, \ldots, m \mid 2, \ldots, n]$ is an SSR matrix. So, the matrix $A^{(2)}[2, \ldots, m \mid 2, \ldots, n]$ is an $(m-1) \times(n-1)$ SSR matrix with $\varepsilon_{1}\left(A^{(2)}[2, \ldots, m \mid 2, \ldots, n]\right)=\varepsilon_{1}(A)$. By the induction hypothesis, for all $k \in\{1, \ldots, r-1\}$, the matrices $A^{(k+1)}[k+1, \ldots, m \mid k+$ $1, \ldots, n]$ are SSR with $\varepsilon_{1}\left(A^{(k+1)}[2, \ldots, m \mid 2, \ldots, n]\right)=\varepsilon_{1}\left(A^{(2)}[2, \ldots, m \mid 2, \ldots, n]\right)(=$ $\left.\varepsilon_{1}(A)\right)$. From this last fact, and taking into account that the first row of $A^{(k)}$ coincides with either the first or the last row of $A$, we conclude that $\varepsilon_{1}\left(A^{(k)}\right)=\varepsilon_{1}(A)$ for all $k$. 


\section{A CHARACTERIZATION OF STRICTLY SIGN REgUlar MATRICES}

In this section, a product with an empty set of indices is by definition 1 and a sum with an empty set of indices is by definition 0 . Thus, it will be useful to denote $A_{l}:=A[1, \ldots, m \mid l, \ldots, n]$. We need the following auxiliary result.

Lemma 3.1. Let $A$ be an $m \times n$ matrix with $m \geq n$. If we apply Neville elimination of $A$ without row exchanges and all the pivots are nonzero, then given $\alpha \in Q_{k, m}$ with $1 \leq \alpha_{1} \leq m-k+1,1 \leq k \leq n$ and $d(\alpha)=0$, we have, for $1 \leq l \leq k$, that

$$
\operatorname{det} A[\alpha \mid 1, \ldots, k]=\left(\prod_{j=1}^{l-1} p_{\alpha_{1}+j-1, j}\right) \operatorname{det} A^{(l)}\left[\alpha_{1}+l-1, \ldots, \alpha_{1}+k-1 \mid l, \ldots, k\right],
$$

where $p_{i j}$ is the $(i, j)$ pivot of the Neville elimination of $A$ for $1 \leq j \leq n$ and $j \leq i \leq m$.

Proof. Let us consider the $k \times k$ submatrix $A[\alpha \mid \beta]$ of $A$, where $\beta=\{1, \ldots, k\}$. If we apply Neville elimination without row exchanges to the submatrix $A[\alpha \mid \beta]$, the pivots that appear are nonzero because they form a subset of the pivots of $A$. Then, for $1 \leq l \leq k, A^{(l)}[\alpha \mid \beta]$ has zeros below its main diagonal in the first $l-1$ columns and the first $l-1$ diagonal elements are the $\left(\alpha_{1}+j-1, j\right)$ pivots $p_{\alpha_{1}+j-1, j}$ of $A$ $(1 \leq j \leq l-1)$. Moreover, since $A^{(l)}[\alpha \mid \beta]$ is obtained from $A[\alpha \mid \beta]$ by performing elementary steps in which we add to a row an appropriate multiple of the previous one, $\operatorname{det} A[\alpha \mid \beta]=\operatorname{det} A^{(l)}[\alpha \mid \beta]$ for all $1 \leq l \leq k$. So, for all $1 \leq l \leq k,(3.1)$ holds.

The next result characterizes SSR matrices through Neville elimination with two-determinant pivoting. Given an $r \times s$ matrix $M$, a column-initial minor of $M$ is a minor of the form $\operatorname{det} M[\alpha \mid 1, \ldots, k]$, where $\alpha \in Q_{k, r}$ with $d(\alpha)=0$ and $1 \leq k \leq \min \{r, s\}$. Analogously, a row-initial minor of $M$ is a minor of the form $\operatorname{det} M[1, \ldots, k \mid \alpha]$, where $\alpha$ and $k$ are given as above. An initial minor of $M$ is either a column-initial or a row-initial minor of $M$.

Theorem 3.2. Let $A$ be an $m \times n(m \geq n)$ matrix. Then $A$ is $S S R$ if and only if we can apply the Neville elimination with two-determinant pivoting to the matrices $A_{l}:=A[1, \ldots, m \mid l, \ldots, n]$ for $l=1, \ldots, n$, all the pivots are nonzero and have the same sign, and the associated permutations occur in steps $k_{i}$, with $k_{i} \leq n-l$, where $k_{1}, \ldots, k_{h}$ are the steps associated to the permutations of the Neville elimination with two-determinant pivoting of $A$.

Proof. Since the matrices $A_{l}$ for all $l=1, \ldots, n$ are submatrices of the SSR matrix $A$, they are also SSR with signature $\left(\varepsilon_{1}, \ldots, \varepsilon_{n-l+1}\right)$. If we apply Proposition 2.1 to each matrix $A_{l}$, we deduce that the matrices appearing through its elimination process $(\operatorname{see}(2.1)), A_{l}^{(j)}[j, \ldots, m \mid l+j-1, \ldots, n]$ and $\tilde{A}_{l}^{(j)}[j, \ldots, m \mid l+j-1, \ldots, n]$ with $j=1, \ldots, n-l+1$, are SSR with the same strict sign of $A$. Then, by (2.3), we conclude that all the pivots of the Neville elimination with two-determinant pivoting of the matrices $A_{l}$ for all $l=1, \ldots, n$ have the same strict sign of $A$.

Now, let us see that the associated permutations to the elimination of the matrices $A_{l}$ for all $l=1, \ldots, n$ occur in the same steps as in $A$. For $l=1, \ldots, n$ and $j=1, \ldots, n-l+1$, we denote by $\left(\varepsilon_{1, l}^{(j)}, \ldots, \varepsilon_{n-l-j+2, l}^{(j)}\right)$ and $\left(\tilde{\varepsilon}_{1, l}^{(j)}, \ldots, \tilde{\varepsilon}_{n-l-j+2, l}^{(j)}\right)$ the associated signatures of the matrices

$$
A_{l}^{(j)}[j, \ldots, m \mid l+j-1, \ldots, n]
$$


and

$$
\tilde{A}_{l}^{(j)}[j, \ldots, m \mid l+j-1, \ldots, n]
$$

respectively.

Let us prove by induction on $j$ that, for $l=1, \ldots, n$,

$$
\varepsilon_{i, l}^{(j)}=\varepsilon_{i, 1}^{(j)}, \quad 1 \leq i \leq n-l-j+2 .
$$

For $j=1$, the result holds, due to the fact that the matrices $A_{l}\left(=A_{l}^{(1)}\right)$ are SSR with signature $\left(\varepsilon_{1}, \ldots, \varepsilon_{n-l+1}\right)\left(=\left(\varepsilon_{1, l}^{(1)}, \ldots, \varepsilon_{n-l+1, l}^{(1)}\right)\right)$ for all $l=1, \ldots, n$. Now, let us assume that (3.2) holds for $j$ and let us prove it for $j+1$. Applying formula (2.4) (taking $C:=\tilde{A}_{l}^{(j)}[j, \ldots, j+i \mid l+j-1, \ldots, l+j-1+i]$ and $\gamma:=(1)$ ) we obtain that, for all $l=1, \ldots, n$, the SSR matrix $A_{l}^{(j+1)}[j+1, \ldots, m \mid l+j, \ldots, n]$ has signature $\left(\varepsilon_{1, l}^{(j+1)}, \ldots, \varepsilon_{n-l-j+1, l}^{(j+1)}\right)$, where

$$
\varepsilon_{i, l}^{(j+1)}=\tilde{\varepsilon}_{i+1, l}^{(j)} / \tilde{\varepsilon}_{1, l}^{(j)}, \quad 1 \leq i \leq n-l-j+1 .
$$

By the induction hypothesis, we know that $\varepsilon_{i, l}^{(j)}=\varepsilon_{i, 1}^{(j)}$ for all $l=1, \ldots, n$ with $i=1, \ldots, n-l-j+2$. So, following the criterion of the pivoting strategy, the matrices $\tilde{A}_{l}^{(j)}$ are obtained from $A_{l}^{(j)}$ in the same way for all $l=1, \ldots, n$. Then, we have that $\tilde{\varepsilon}_{i, l}^{(j)}=\tilde{\varepsilon}_{i, 1}^{(j)}$ for all $l=1, \ldots, n$ with $i=1, \ldots, n-l-j+2$ and so, by (3.3), $\varepsilon_{i, l}^{(j+1)}=\tilde{\varepsilon}_{i+1,1}^{(j)} / \tilde{\varepsilon}_{1,1}^{(j)}=\varepsilon_{i, 1}^{(j+1)}$, and the induction holds for $j+1$.

Taking $i=2$ in (3.2), we conclude that, for all $l=1, \ldots, n$, the minors of order 2 of the matrices $A_{l}^{(j)}[j, \ldots, m \mid l+j-1, \ldots, n]$ have the same strict sign $\varepsilon_{2,1}^{(j)}$. So, the associated permutations to the Neville elimination with two-determinant pivoting of the matrices $A_{l}$ for all $l=1, \ldots, n$ occur in the same steps as $A$.

Next we prove that the condition is sufficient for the strict sign regularity of $A$. We suppose that the Neville elimination with two-determinant pivoting of a rectangular matrix, $B=\left(b_{i j}\right)_{1 \leq j \leq v}^{1 \leq j \leq u}(u \geq v)$, is applied with all the pivots of the same strict sign $\delta$. Let $r(0 \leq r \leq v-1)$ be the number of permutations needed through the Neville elimination with two-determinant pivoting of $B$ and let $k_{1}, \ldots, k_{r}$ be the steps of this elimination associated with the previous permutations. We define $k_{r+1}:=v+1$ and $k_{0}:=0$. We note that given any positive integer $d$ with $1 \leq d \leq v$, there exists a unique $j \in\{0, \ldots, r\}$ such that $k_{j} \leq d \leq k_{j+1}-1$. Now, let us prove by induction on $r$ that all the column-initial minors of $B$ of order $d$ have the following strict sign:

$$
(-1)^{\sum_{t=1}^{j}\left\lfloor\frac{d-k_{t}+1}{2}\right\rfloor} \cdot \delta^{d}, \quad k_{j} \leq d \leq k_{j+1}-1, \quad j \in\{0, \ldots, r\},
$$

where given a positive real number $x,\lfloor x\rfloor$ denotes the greatest integer less than or equal to $x$.

Let us first suppose that $r=0$, that is, Neville elimination of $B$ with twodeterminant pivoting does not use row exchanges. In this case, the associated $j=0$ for all $d$. We can observe that the $(i, 1)$ pivots of this elimination procedure are $p_{i 1}=b_{i 1}$ for all $i=1, \ldots, u$. So the column-initial minor of order 1 of the matrix $B$ have the same strict sign $\delta$, and (3.4) holds for $d=1$. Let us define the increasing sequence of $s(1 \leq s \leq v)$ consecutive positive integers $\alpha_{i, s}:=\{i-s+1, \ldots, i\}$ with $s \leq i \leq u$. 
Taking into account that if we apply Neville elimination to $B\left[\alpha_{i, d} \mid 1, \ldots, d\right]$ no row exchanges are needed and that the pivots are nonzero since they are also pivots of the matrix $B$, we can apply Lemma 3.1 to the submatrix $B\left[\alpha_{i, d} \mid 1, \ldots, d\right]$ of $B$ and we have

$$
\operatorname{det} B\left[\alpha_{i, d} \mid 1, \ldots, d\right]=\prod_{k=1}^{d} p_{i-d+k, k}, \quad i=d, \ldots, u .
$$

Since all the pivots of the matrix $B$ are nonzero and have the same strict sign $\delta$, we deduce from (3.5) that all the column-initial minors of order $d(2 \leq d \leq v)$ of the matrix $B$ have the same strict $\operatorname{sign} \delta^{d}$ and (3.4) holds for $2 \leq d \leq \bar{v}$.

Now, let us assume that the induction holds for $r-1$ and let us prove it for $r$. Let $B$ be a matrix such that Neville elimination with two-determinant pivoting requires $r$ row exchanges.

Analogously to the case $r=0$, we can prove for any $d$ with $1 \leq d \leq k_{1}-1$ (if $\left.k_{1}>1\right)$ that all the column-initial minors of order $d$ of the matrix $B$ have the same strict $\operatorname{sign} \delta^{d}$.

Taking into account the criterion of two-determinant pivoting strategy, we know that

$$
\tilde{B}^{\left(k_{1}\right)}\left[k_{1}, \ldots, u \mid k_{1}, \ldots, v\right]=P_{k_{1}} B^{\left(k_{1}\right)}\left[k_{1}, \ldots, u \mid k_{1}, \ldots, v\right]
$$

with $P_{k_{1}}$ the backward identity of order $u-k_{1}+1$.

If $k_{1} \neq 1$, then we can apply the Neville elimination of $B\left[\alpha_{i, d} \mid 1, \ldots, d\right]$ without row exchanges up to the $k_{1}$-th step and with all the pivots nonzero since they are pivots of the matrix $B$. Then, applying Lemma 3.1 to the submatrix $B\left[\alpha_{i, d} \mid 1, \ldots, d\right]$ of $B$, we can write the following formula:

$\operatorname{det} B\left[\alpha_{i, d} \mid 1, \ldots, d\right]=\left(\prod_{k=1}^{k_{1}-1} p_{i-d+k, k}\right) \operatorname{det} B^{\left(k_{1}\right)}\left[\alpha_{i, d-k_{1}+1} \mid k_{1}, \ldots, d\right], \quad i=d, \ldots, u$.

Observe that the previous formula (3.7) trivially holds for $k_{1}=1$. By (3.6) we can rewrite $(3.7)$ as

$\operatorname{det} B\left[\alpha_{i, d} \mid 1, \ldots, d\right]=(-1)^{\left\lfloor\frac{d-k_{1}+1}{2}\right\rfloor}\left(\prod_{k=1}^{k_{1}-1} p_{i-d+k, k}\right) \operatorname{det} \tilde{B}^{\left(k_{1}\right)}\left[\alpha_{u-i+d, d-k_{1}+1} \mid k_{1}, \ldots, d\right]$,

for $i=d, \ldots, u$.

Let us define $\tilde{k}_{t}:=k_{t+1}-\left(k_{1}-1\right)$ for $t \in\{0, \ldots, r\}$. By our hypothesis, Neville elimination with two-determinant pivoting of the rectangular matrix $\tilde{B}^{\left(k_{1}\right)}\left[k_{1}, \ldots, u \mid k_{1}, \ldots, v\right]$ has $r-1$ permutations associated with the steps $\tilde{k}_{1}, \ldots, \tilde{k}_{r-1}$. Moreover, we can observe that $\operatorname{det} \tilde{B}^{\left(k_{1}\right)}\left[\alpha_{u-i+d, d-k_{1}+1} \mid k_{1}, \ldots, d\right]$ is a column-initial minor of order $\tilde{d}:=d-k_{1}+1$ of the matrix $\tilde{B}^{\left(k_{1}\right)}\left[k_{1}, \ldots, u \mid k_{1}, \ldots, v\right]$. Taking into account that given any $d$, with $k_{1} \leq d \leq v$, there exists a unique $j \in\{1, \ldots r\}$ such that $k_{j} \leq d \leq k_{j+1}-1$, we can conclude that there exists a unique $j \in\{0, \ldots, r-1\}$ such that $\tilde{k}_{j} \leq \tilde{d} \leq \tilde{k}_{j+1}-1$. Moreover, the pivots of the matrix $\tilde{B}^{\left(k_{1}\right)}\left[k_{1}, \ldots, u \mid k_{1}, \ldots, v\right]$ are also pivots of the matrix $B$, so that they are nonzero and have the same strict $\operatorname{sign} \delta$. So, the matrix $\tilde{B}^{\left(k_{1}\right)}\left[k_{1}, \ldots, u \mid k_{1}, \ldots, v\right]$ satisfaces the induction hypothesis and then we have that all the column-initial minors of the matrix $\tilde{B}^{\left(k_{1}\right)}\left[k_{1}, \ldots, u \mid k_{1}, \ldots, v\right]$ have the same strict sign that is given 
by (3.4):

$$
(-1)^{\sum_{t=1}^{j-1}\left\lfloor\frac{\tilde{d}-\tilde{k}_{t}+1}{2}\right\rfloor} \cdot \delta^{\tilde{d}}, \quad \tilde{k}_{j} \leq \tilde{d} \leq \tilde{k}_{j+1}-1, \quad j \in\{0, \ldots, r-1\} .
$$

Now, taking into account the definition of $\tilde{k}_{t}$ and $\tilde{d}$, we can write

$$
\operatorname{sign}\left(\operatorname{det} \tilde{B}^{\left(k_{1}\right)}\left[\alpha_{u-i+d, d-k_{1}+1} \mid k_{1}, \ldots, d\right]\right)=(-1)^{\sum_{t=2}^{j}\left\lfloor\frac{d-k_{t}+1}{2}\right\rfloor} \cdot \delta^{d-k_{1}+1}
$$

for $k_{j} \leq d \leq k_{j+1}-1, \quad j \in\{1, \ldots, r\}$.

Finally, from (3.9) and (3.8), and taking into account the obtained result for the column-initial minors of order $d$ with $1 \leq d \leq k_{1}-1$, it follows that the sign of all column-initial minors of order $d$, with $1 \leq d \leq v$, of the matrix $B$ is given by (3.4) and it is strict. So, the induction holds.

Now, since all matrices $A_{l}$ for all $l=1, \ldots, n$ satisfy the hypothesis of $B$ and, by our hypothesis on $A_{l}$, their associated permutations occur in steps $k_{i}$, with $k_{i} \leq$ $n-l$, we have that all column-initial minors of the matrices $A_{l}$ for all $l=1, \ldots, n$ have the same strict sign given by (3.4). Taking into account that any minor of $A$ with consecutive rows and columns is a column-initial minor of some matrix $A_{l}$ with $l=1, \ldots, n$, we conclude that the minors of $A$ using $d(1 \leq d \leq n)$ consecutive rows and columns have the same strict sign $\varepsilon_{d}$. Then, by Theorem 2.5 of [1], $A$ is a strictly sign regular matrix with signature $\varepsilon=\left(\varepsilon_{1}, \ldots, \varepsilon_{n}\right)$.

\section{A test For StRICT Sign REgUlarity}

In this section, we provide a test to check the strict sign regularity of a matrix $A$.

Remark 4.1. By Proposition 2.1, if $A=\left(a_{i j}\right)_{1 \leq i \leq m}^{1 \leq j \leq n}(m \geq n)$ is an SSR matrix with signature $\left(\varepsilon_{1}, \varepsilon_{2}, \ldots, \varepsilon_{n}\right)$, then all the matrices $A^{(k)}[k, \ldots, m \mid k, \ldots, n]$, appearing through Neville elimination with two-determinant pivoting of $A$, are SSR with strict sign $\varepsilon_{1}$. Thus, $-A=\left(-a_{i j}\right)_{1 \leq j \leq m}^{\substack{1 \leq j \leq n \\ 1 \leq i \leq m}}$ is an SSR matrix with signature $\left(-\varepsilon_{1}, \varepsilon_{2}, \ldots,(-1)^{n} \varepsilon_{n}\right)$ and all the matrices $-A^{(k)}[k, \ldots, m \mid k, \ldots, n]$, appearing through Neville elimination with two-determinant pivoting of $-A$, are SSR with strict $\operatorname{sign}-\varepsilon_{1}$. So, the associated permutations to Neville elimination with two-determinant pivoting of $A$ and $-A$ occur in the same steps, and the pivots of $-A$ are opposite in sign to those of $A$. On the other hand, if we consider $P$, the backward identity matrix of order $m$, then $P A$ is an SSR matrix with signature $\left(\varepsilon_{1},-\varepsilon_{2}, \ldots,(-1)^{\left\lfloor\frac{n}{2}\right\rfloor} \varepsilon_{n}\right)$, and Neville elimination with two-determinant pivoting of $A$ and $P A$ only differs in the first step, where either $A$ or $P A$ needs a row exchange. Finally, $A^{T}$ is an $n \times m$ SSR matrix with the same signature as $A$. Taking into account formula (2.4), it can be seen that the permutations of Neville elimination with two-determinant of $A$ and $A^{T}$ occur in the same steps and all the pivots have the same strict $\operatorname{sign} \varepsilon_{1}$.

The next result characterizes totally positive matrices through Neville elimination with two-determinant pivoting.

Proposition 4.2. Let $A$ be an $m \times n$ matrix. Then the following conditions are equivalent:

(1) A is totally positive.

(2) Neville elimination with two-determinant pivoting of $A$ and $A^{T}$ can be carried out without row exchanges, and all the pivots are positive. 
Proof. (1) $\Rightarrow(2)$ Applying the proof of Proposition 2.1 to a totally positive matrix $A$, we obtain that $\tilde{A}^{(1)}=A^{(1)}$ and $A^{(2)}[2, \ldots, m \mid 2, \ldots, n]$ is totally positive; then it can be proved by induction that all the matrices $A^{(k)}[k, \ldots, m \mid k, \ldots, n]$ are totally positive. Then, we do not need row exchanges through Neville elimination with two-determinant pivoting of $A$, and all the pivots are positive. By Remark 4.1, we obtain the result for $A^{T}$, and (2) follows.

(2) $\Rightarrow$ (1) By applying formula (3.5) to $B=A$ and to $B=A^{T}$, we obtain that the column-initial minors of $A$ and $A^{T}$ are positive. Then, by Theorem 4.1 of $[6$, $A$ is totally positive.

Taking into account the Schur complement formula (2.4), one can easily deduce that all pivots and multipliers of Gauss elimination of a totally positive matrix $A$ are positive (and so, also those of $A_{l}:=A[1, \ldots, m \mid l, \ldots, n]$ for all $l=1, \ldots, n$ and of their transposes). However the following example shows that their positivity does not imply the total positivity of $A$. This illustrates the convenience of using Neville elimination when dealing with these matrices (and, more generally, with SSR matrices).

Example 4.3. The matrix

$$
A=\left(\begin{array}{lll}
1 & 2 & 1 \\
1 & 4 & 5 \\
1 & 3 & 4
\end{array}\right)
$$

is not totally positive because $\operatorname{det} A[2,3 \mid 1,2]<0$. Let us denote by $p_{i j}^{(l)}$ (resp., $q_{i j}^{(l)}$ ) and $m_{i j}^{(l)}$ (resp., $n_{i j}^{(l)}$ ) the pivots and multipliers of Gauss elimination of $A_{l}$ (resp., $\left(A_{l}\right)^{T}$ ). Gauss elimination of $A=A_{1}$ (resp., $A^{T}=\left(A_{1}\right)^{T}$ ) produces an upper triangular matrix $U$ (resp., $V$ ):

$$
U=\left(\begin{array}{lll}
1 & 2 & 1 \\
0 & 2 & 4 \\
0 & 0 & 1
\end{array}\right), \quad\left(\text { resp. }, \quad V=\left(\begin{array}{lll}
1 & 1 & 1 \\
0 & 2 & 1 \\
0 & 0 & 1
\end{array}\right)\right)
$$

by means of the positive pivots $p_{11}^{(1)}=1, p_{22}^{(1)}=2, p_{33}^{(1)}=1$ (resp., $q_{11}^{(1)}=1, q_{22}^{(1)}=$ $2, q_{33}^{(1)}=1$ ) and multipliers $m_{21}^{(1)}=m_{31}^{(1)}=1, m_{32}^{(1)}=1 / 2$ (resp., $n_{21}^{(1)}=2, n_{31}^{(1)}=$ $1, n_{32}^{(1)}=2$ ). Gauss elimination of $A_{2}$ (resp., $\left.\left(A_{2}\right)^{T}\right)$ has positive pivots $p_{11}^{(2)}=$ $2, p_{22}^{(2)}=3\left(\right.$ resp., $q_{11}^{(2)}=2, q_{22}^{(2)}=3$ ) and multipliers $m_{21}^{(2)}=2, m_{31}^{(2)}=3 / 2, m_{32}^{(2)}=$ $5 / 6$ (resp., $n_{21}^{(2)}=1 / 2$ ). Gauss elimination of $A_{3}$ (resp., $\left(A_{3}\right)^{T}$ ) has positive pivots $p_{11}^{(3)}=1$ (resp., $q_{11}^{(3)}=1$ ) and multipliers $m_{21}^{(3)}=5, m_{31}^{(3)}=4$. Observe that, for the matrix $A$, Gauss elimination coincides with Gauss elimination with partial pivoting. In contrast, after applying one step of Neville elimination of $A$ with two-determinant pivoting (and so, without row exchanges), we deduce that $A$ is not totally positive because we obtain a negative pivot $p_{32}=-1$.

Taking into account Remark 4.1 and Proposition 4.2 we can derive the following results.

Proposition 4.4. Let $A$ be an $m \times n$ matrix. Then the following conditions are equivalent:

(1) $-A$ is totally positive.

(2) Neville elimination with two-determinant pivoting of $A$ and $A^{T}$ can be carried out without row exchanges, and all the pivots are negative. 
Proposition 4.5. Let $A$ be an $m \times n$ matrix and let $P$ be the backward identity matrix of order $m$. Then the following conditions are equivalent:

(1) $P A$ (resp., $-P A)$ is totally positive.

(2) Neville elimination with two-determinant pivoting of $A$ and $A^{T}$ only needs one row exchange in the first step, and all the pivots are positive (resp., negative).

The next result characterizes totally negative matrices through Neville elimination with two-determinant pivoting.

Proposition 4.6. Let $A$ be an $m \times n$ matrix with $m \geq n$. Then the following conditions are equivalent:

(1) A is totally negative.

(2) The $(i, n)$ entries, $i=n, \ldots, m$, of $A$ are negative, and we can apply the Neville elimination with two-determinant pivoting of $A$ and $A^{T}$, needing only two row exchanges associated to the first and the second steps, and all the pivots are negative.

Proof. First we prove that the condition is necessary. Since $A$ is totally negative, it is obvious that $a_{i n}<0$ for all $i=n, \ldots, m$. Moreover, the first step of Neville elimination with two-determinant pivoting of $A$ needs a row exchange obtaining $\tilde{A}^{(1)}:=P_{1} A$, where $P_{1}$ is the backward identity matrix of order $m$. By Remark 4.1, $\tilde{A}^{(1)}$ is SSR with signature $\left(\tilde{\varepsilon}_{1}^{(1)}, \ldots, \tilde{\varepsilon}_{n}^{(1)}\right)$, where $\tilde{\varepsilon}_{k}^{(1)}=(-1)^{\left\lfloor\frac{k}{2}\right\rfloor+1}$. By Proposition $2.1, A^{(2)}[2, \ldots, m \mid 2, \ldots, n]$ is SSR with signature $\left(\varepsilon_{1}^{(2)}, \ldots, \varepsilon_{n-1}^{(2)}\right)$ and $\varepsilon_{1}^{(2)}=\varepsilon_{1}=$ -1. By formula (2.4) (taking $C:=\tilde{A}^{(1)}[1, \alpha \mid 1, \beta]$ and $\gamma=(1)$, with $\alpha \in Q_{k, m}$, $\beta \in Q_{k, n}, 1<\alpha_{1}, \beta_{1}$, and $\left.1 \leq k \leq n-1\right)$, we have $\varepsilon_{k}^{(2)}=\tilde{\varepsilon}_{k+1}^{(1)} / \tilde{\varepsilon}_{1}^{(1)}=(-1)^{\left\lfloor\frac{k+1}{2}\right\rfloor}$ with $k=1, \ldots, n-1$. Since $\varepsilon_{2}^{(2)}=-1$, we need a row exchange for obtaining $\tilde{A}^{(2)}[2, \ldots, m \mid 2, \ldots, n]=P_{2} A^{(2)}[2, \ldots, m \mid 2, \ldots, n]$, where $P_{2}$ is the backward identity matrix of order $m-1$. By Remark $4.1, \tilde{A}^{(2)}[2, \ldots, m \mid 2, \ldots, n]$ is SSR with signature $\left(\tilde{\varepsilon}_{1}^{(2)}, \ldots, \tilde{\varepsilon}_{n-1}^{(2)}\right)$, where $\tilde{\varepsilon}_{k}^{(2)}=(-1)^{\left\lfloor\frac{k+1}{2}\right\rfloor+\left\lfloor\frac{k}{2}\right\rfloor}=(-1)^{k}$. Then, the matrix $-\tilde{A}^{(2)}[2, \ldots, m \mid 2, \ldots, n]$ is totally positive and, by Proposition 4.4 , the Neville elimination with two-determinant pivoting of $\tilde{A}^{(2)}[2, \ldots, m \mid 2, \ldots, n]$ does not need row exchanges, and all the pivots are negative. The result for $A^{T}$ also follows from Remark 4.1.

Now we prove that the condition is sufficient. Applying formula (3.4) (taking $r=2, \delta=-1, k_{1}=1$ and $k_{2}=2$ ) to $A$ and $A^{T}$, we obtain that the initial minors of $A$ are negative. Now, for $t=1, \ldots, m-n+1$, let us prove by induction on $t$ that $A[t, \ldots, n+t-1 \mid 1, \ldots, n]$ is totally negative. If $t=1$, we note that the initial minors of any order of $A[1, \ldots, n]$ are also initial minors of $A$, which are negative. Then, by Remark 3.6 of $[7$, we have that $A[1, \ldots, n]$ is totally negative, and the result holds for $t=1$. Let us assume that it holds for $t-1$, and let us prove it for $t$. By the induction hypothesis, $A[t-1, \ldots, n+t-2 \mid 1, \ldots, n]$ is totally negative. In consequence, all the row-initial minors of order not greater than $n-1$ of $A[t, \ldots, n+t-1 \mid 1, \ldots, n]$ are negative. The column-initial minors of any order of $A[t, \ldots, n+t-1 \mid 1, \ldots, n]$ (one of them being the whole determinant) are also column-initial minors of $A$, and so are negative. So, by Remark 3.6 of [7, we have that $A[t, \ldots, n+t-1 \mid 1, \ldots, n]$ is totally negative, and the induction holds for $t$.

Taking into account that any minor of $A$ with consecutive rows and columns is a minor of some totally negative matrix $A[t, \ldots, n+t-1 \mid 1, \ldots, n]$ with $t=$ 
$1, \ldots, n+t-1$, we can conclude that the minors of $A$ using $d(1 \leq d \leq n)$ consecutive rows and columns are negative. Then, by Theorem 2.5 of [1], $A$ is totally negative.

Remark 4.1 and Proposition 4.6 allow us to derive the following results.

Proposition 4.7. Let $A$ be an $m \times n$ matrix with $m \geq n$. Then the following conditions are equivalent:

(1) $-A$ is totally negative.

(2) The entries $(i, n), i=n, \ldots, m$, of $A$ are positive, and we can apply the Neville elimination with two-determinant pivoting of $A$ and $A^{T}$, needing only two row exchanges associated to the first and the second steps, and all the pivots are positive.

Proposition 4.8. Let $A$ be an $m \times n$ matrix with $m \geq n$ and let $P$ be the backward identity matrix of order $m$. Then the following conditions are equivalent:

(1) PA (resp., $-P A)$ is totally negative.

(2) The entries $(i, n), i=1, \ldots, m-n+1$, of $A$ are negative (resp., positive) and we can apply the Neville elimination with two-determinant pivoting of $A$ and $A^{T}$, needing only one row exchange associated to the second step, and all the pivots are negative (resp., positive).

The following result provides a test to check if a matrix is SSR. In the Introduction we have commented on many problems where such a test can be useful.

Theorem 4.9. Let $A$ be an $m \times n(m \geq n)$ matrix. Let $A_{l}:=A[1, \ldots, m \mid l, \ldots, n]$ for $l=1, \ldots, n$. Then $A$ is $S S R$ if and only if we can apply the Neville elimination with two-determinant pivoting of $A$ (and then the associated permutations occur in steps $k_{1}, \ldots, k_{h}$ ) and the following conditions hold:

(1) All the pivots of Neville elimination with two-determinant pivoting of the matrix $A=A_{1}$ are nonzero with sign $\varepsilon_{1}$.

(2) For all $i=1, \ldots, m-n+1$ and $i=n, \ldots m, \varepsilon_{1} a_{i n}>0$.

(3) a) If Neville elimination with two-determinant pivoting of $A$ does not use permutations, then we can also apply the same elimination of $A^{T}$, it does not use permutations and all its pivots are nonzero with sign $\varepsilon_{1}$.

b) If $k_{1}>2$, then we can apply the Neville elimination with two-determinant pivoting of the matrices $A_{l}$ for $l=2, \ldots, n-k_{1}+1$, the associated permutations occur in steps $k_{i} \leq n-l$ and $\left(A_{n-k_{1}+1}\right)^{T}$ does not need row exchanges. Moreover, all their pivots are nonzero with sign $\varepsilon_{1}$.

c) If $k_{1} \leq 2$ and $h=1$, then we can apply the Neville elimination of $A^{T}$ with two-determinant pivoting, only one permutation occurs in step $k_{1}$ and all its pivots are nonzero with sign $\varepsilon_{1}$.

d) If $k_{1}=2$ and $h>1$, then we can apply the Neville elimination with two-determinant pivoting of the matrices $A_{l}$ for $l=2, \ldots, n-k_{2}+1$ and $\left(A_{n-k_{2}+1}\right)^{T}$, and the associated permutations occur in steps $k_{i} \leq$ $n-l$ and $k_{1}$, respectively. Moreover, all their pivots are nonzero with $\operatorname{sign} \varepsilon_{1}$.

e) If $k_{1}=1$ and $k_{2}>2$, then we can apply the Neville elimination with two-determinant pivoting of the matrices $A_{l}$ for $l=2, \ldots, n-k_{2}+1$ and $\left(A_{n-k_{2}+1}\right)^{T}$, and the associated permutations occur in steps $k_{i} \leq$ $n-l$ and $k_{1}$, respectively. Moreover, all their pivots are nonzero with sign $\varepsilon_{1}$. 
f) If $k_{1}=1, k_{2}=2$ and $h=2$, then we can apply the Neville elimination of $A^{T}$ with two-determinant pivoting, and only two permutations occur in steps $k_{1}$ and $k_{2}$. Moreover, all its pivots are nonzero with sign $\varepsilon_{1}$.

g) If $k_{1}=1, k_{2}=2$ and $h>2$, then we can apply the Neville elimination with two-determinant pivoting of the matrices $A_{l}$ for $l=2, \ldots, n-$ $k_{3}+1$ and $\left(A_{n-k_{3}+1}\right)^{T}$, and the associated permutations occur in steps $k_{i} \leq n-l$ and $k_{1}, k_{2}$, respectively. Moreover, all their pivots are nonzero with sign $\varepsilon_{1}$.

Proof. If $A$ is SSR, then (1) holds by Theorem 3.2 and (2) is obvious. Since the submatrices $A_{l}$ are also SSR, again by Theorem 3.2, (3) follows.

Conversely, assume that (1), (2), and (3) hold. If permutations are not needed, by Propositions 4.2 and 4.4, either $A$ or $-A$ is totally positive. If $k_{1}=1$ and $h=1$, then, by Proposition 4.5, either $P A$ or $-P A$ is totally positive. If $k_{1}=2$ and $h=1$, then, by Proposition 4.8 , either $P A$ or $-P A$ is totally negative. If $k_{1}=1, k_{2}=2$ and $h=2$, by Propositions 4.6 and 4.7 , either $A$ or $-A$ is totally negative. Hence, in cases a), c) and f), we have already proved that $A$ is SSR and so the converse holds.

If $k_{1}>2$, by (3), the Neville elimination with two-determinant pivoting of $A_{n-k_{1}+1}$ (resp., $\left(A_{n-k_{1}+1}\right)^{T}$ ) can be carried out without row exchanges and all their pivots are nonzero with sign $\varepsilon_{1}$. So, by Propositions 4.2 and 4.4, either $A_{n-k_{1}+1}$ or $-A_{n-k_{1}+1}$ is totally positive. Then, for $l=n-k_{1}+2, \ldots, n$, since $A_{l}$ or $-A_{l}$ are submatrices of $A_{n-k_{1}+1}$ or $-A_{n-k_{1}+1}$ respectively, they are also totally positive.

If $k_{1}=2$ and $h>1$, by (3), the Neville elimination with two-determinant pivoting of $A_{n-k_{2}+1}$ (resp., $\left(A_{n-k_{2}+1}\right)^{T}$ ) can be carried out with one row exchange associated to the second step and all their pivots are nonzero with sign $\varepsilon_{1}$. So, by Proposition 4.8 , either $P A_{n-k_{2}+1}$ or $-P A_{n-k_{2}+1}$ is totally negative. Then, for $l=n-k_{2}+2, \ldots, n$, since $P A_{l}$ or $-P A_{l}$ are submatrices of $P A_{n-k_{2}+1}$ or $-P A_{n-k_{2}+1}$, respectively, they are also totally negative.

If $k_{1}=1$ and $k_{2}>2$, by (3), the Neville elimination with two-determinant pivoting of $A_{n-k_{2}+1}$ (resp., $\left(A_{n-k_{2}+1}\right)^{T}$ ) can be carried out with one row exchange associated to the first step and all their pivots are nonzero with sign $\varepsilon_{1}$. So, by Proposition 4.5, either $P A_{n-k_{2}+1}$ or $-P A_{n-k_{2}+1}$ is totally positive. Then, for $l=$ $n-k_{2}+2, \ldots, n$, since $P A_{l}$ or $-P A_{l}$ are submatrices of $P A_{n-k_{2}+1}$ or $-P A_{n-k_{2}+1}$, respectively, they are also totally positive.

If $k_{1}=1$ and $k_{2}=2$ and $h>2$, by (3), the Neville elimination with twodeterminant pivoting of $A_{n-k_{3}+1}$ (resp., $\left(A_{n-k_{3}+1}\right)^{T}$ ) can be carried out with two row exchanges associated to the first and the second step and all their pivots are nonzero with sign $\varepsilon_{1}$. So, by Proposition 4.6 and Proposition 4.7, either $A_{n-k_{3}+1}$ or $-A_{n-k_{3}+1}$ is totally negative. Then, for $l=n-k_{3}+2, \ldots, n$, since $A_{l}$ or $-A_{l}$ are submatrices of $A_{n-k_{3}+1}$ or $-A_{n-k_{3}+1}$, respectively, they are also totally negative.

In all remaining cases b), d), e) and g), when we apply Neville elimination with two-determinant pivoting to the matrices $A_{l}$ for $l=1, \ldots, n$, all the pivots are nonzero and have sign $\varepsilon_{1}$ and the associated permutations occur in the steps $k_{i}$ with $k_{i} \leq n-l$. So, by Theorem $3.2 A$ is SSR. 


\section{Computational cost}

This section deals with the computational cost of the previous test defined by Theorem 4.9. Given an $m \times n(m \geq n)$ matrix $A$, this test checks if $A$ is SSR performing the Neville elimination with two-determinant pivoting of some submatrices of $A$.

Let us start by analyzing the computational cost of the Neville elimination with two-determinant pivoting of one matrix $A=\left(a_{i j}\right)_{1 \leq i \leq m}^{1 \leq j \leq n}$ with $m \geq n$.

Considering as an arithmetic floating point operation any of the following operations:,,$+- /$ or $*$. For each step $k(1 \leq k \leq n-1)$ we have to carry on one substraction and two multiplications applying two-determinant pivoting strategy, and $(m-k)(n-k+1)$ substractions, $(m-k)(n-k+1)$ multiplications and $(m-k)$ quotients applying the Neville elimination process. Therefore, the computational cost of the Neville elimination with two-determinant determinant pivoting of $A$, denoted by $C_{n}$, is

$$
C_{n}:=\left(\sum_{k=1}^{n-1}(m-k)(2(n-k)+1)\right)+3(n-1) \approx m n^{2}-\frac{n^{3}}{3}
$$

arithmetic floating point operations.

In particular, Neville elimination with two-determinant pivoting of an $n \times n$ matrix requires approximately $2 n^{3} / 3$ arithmetic floating point operations. In fact, Neville elimination requires the same cost as Gauss elimination (approximately $2 n^{3} / 3$ arithmetic floating point operations), and two-determinant pivoting requires $n-1$ additional substractions and $2 n-2$ additional multiplications.

If the $m \times n(m \geq n)$ matrix $A$ belongs to one of the important classes of matrices considered in Proposition 4.2 and Propositions 4.4-4.8, by (5.1) the computational cost of our test will be bounded by approximately $2 \mathrm{~m}^{3} / 3$ arithmetic floating point operations. In general, by Theorem 4.9, we can observe that we have to apply the Neville elimination with two-determinant pivoting strategy at most to $n$ matrices $A_{1}, \ldots, A_{n}$ of orders $m \times n, m \times(n-1), \ldots, m \times 1$, respectively, for checking if $A$ is an SSR matrix. So, the maximum computational cost of the test, denoted by $C^{n}$, will be computed as follows:

$$
C^{n}:=\sum_{r=1}^{n} C_{n-r+1}
$$

where $C_{n-r+1}$ is by $(5.1)$

$$
C_{n-r+1} \approx m n^{2}-\frac{n^{3}}{3}-2 m n r+n^{2} r+m r^{2}-n r^{2}+\frac{r^{3}}{3} .
$$

Taking into account (5.2) it can be checked that the approximate number of arithmetic floating point operations is

$$
C^{n} \approx\left(m n^{2}-\frac{n^{3}}{3}\right) n+\left(\frac{n^{2}-2 m n}{2}\right) n^{2}-\left(\frac{m-n}{3}\right) n^{3}+\frac{n^{4}}{12} .
$$

Observe that, if $m=n$, then $C^{n}$ is bounded above approximately by $n^{4} / 4$.

Now, taking into account a)-g) of Theorem 4.9, we observe that we only have to apply the Neville elimination with two-determinant pivoting strategy to $l$ matrices $(1 \leq l \leq n) A_{1}, \ldots, A_{l}$ of orders $m \times n, m \times(n-1), \ldots, m \times(n-l+1)$, respectively, 
for checking if $A$ is an SSR matrix. So, the computational cost of the test, denoted in this case by $C^{l}$, will be computed as follows:

$$
C^{l}:=\sum_{r=1}^{l} C_{n-r+1},
$$

where $C_{n-r+1}$ is given by (5.2).

Taking into account (5.2) it can be checked that the approximate number of arithmetic floating point operations is

$$
C^{l} \approx\left(m n^{2}-\frac{n^{3}}{3}\right) l+\left(\frac{n^{2}-2 m n}{2}\right) l^{2}-\left(\frac{m-n}{3}\right) l^{3}+\frac{l^{4}}{12} .
$$

\section{Growth Factor and Finite Precision}

Let $A$ be an $m \times n$ matrix. The growth factor associated to Neville elimination with two-determinant pivoting is given by

$$
\rho^{*}(A)=\frac{\max _{i, j, k}\left|a_{i j}^{(k)}\right|}{\max _{i, j}\left|a_{i j}\right|},
$$

with $A^{(k)}=\left(a_{i j}^{(k)}\right)_{1 \leq i \leq m}^{1 \leq j \leq n}$ of the sequence (2.1).

The following result shows that this growth factor is 1 when $A$ is SSR.

Theorem 6.1. If $A$ is an $m \times n$ SSR matrix, then the growth factor (6.1) corresponding to Neville elimination with two-determinant pivoting is optimal:

$$
\rho^{*}(A)=1 .
$$

Proof. Let $r:=\min \{m, n\}$. It is sufficient to see that

$$
\left|a_{i j}^{(t+1)}\right| \leq\left|\tilde{a}_{i j}^{(t)}\right|, \quad t+1 \leq i \leq m, \quad t+1 \leq j \leq n, \quad 1 \leq t \leq r-1 .
$$

By Proposition 2.1 we know that $\varepsilon_{1}(A)=\varepsilon_{1}\left(A^{(t)}\right)\left(=\varepsilon_{1}\left(\tilde{A}^{(t)}\right)\right)$ for all $t \geq 1$. This implies by (2.2) that

$$
\operatorname{sign}\left(\tilde{a}_{i j}^{(t)}\right)=\operatorname{sign}\left(\frac{\tilde{a}_{i t}^{(t)}}{\tilde{a}_{i-1, t}^{(t)}} \tilde{a}_{i-1, j}^{(t)}\right)=\operatorname{sign}\left(a_{i j}^{(t+1)}\right) .
$$

Now, taking into account that

$$
a_{i j}^{(t+1)}=\tilde{a}_{i j}^{(t)}-\left(\tilde{a}_{i t}^{(t)} / \tilde{a}_{i-1, t}^{(t)}\right) \tilde{a}_{i-1, j}^{(t)}
$$

(6.3) follows from (6.4).

Obviously, the growth factor (6.2) avoids overflows in practical computations. In addition, let us recall that the growth factor is an indicator of the stability of an elimination process. The following example shows, in this sense, a clear advantage of the two-determinant pivoting in Neville elimination with respect to Neville elimination without pivoting (as it was used in [7]).

Example 6.2. Let $\varepsilon$ be a positive real number and let $A$ be the SSR matrix given by

$$
A=\left(\begin{array}{ll}
\varepsilon & 1 \\
1 & 1
\end{array}\right)
$$


If we carry out Neville elimination without pivoting and Neville elimination with two-determinant pivoting, we obtain the upper triangular matrices $U$ and $V$, respectively:

$$
U=\left(\begin{array}{cc}
\varepsilon & 1 \\
0 & 1-\frac{1}{\varepsilon}
\end{array}\right), \quad V=\left(\begin{array}{cc}
1 & 1 \\
0 & 1-\varepsilon
\end{array}\right)
$$

Then the growth factor associated to Neville elimination with two-determinant pivoting of $A$ is $\rho^{*}(A)=1$, in contrast to the corresponding one associated to Neville elimination without pivoting, which is $\frac{1}{\varepsilon}-1$, and so can be arbitrarily large.

Now, we analyze the computed sequence of matrices $\hat{A}^{(t)}$ obtained when we apply Neville elimination with two-determinant pivoting of $A$ with finite precision arithmetic.

Proposition 6.3. Let $A$ be an $m \times n S S R$ matrix and $r:=\min \{m, n\}$. Let us assume that when applying Neville elimination with two-determinant pivoting, the associated permutations appear at steps $k_{1}, \ldots, k_{h}$ and all pivots have the same strict sign of $A$. Then, for floating point arithmetic of sufficiently high precision, the Neville elimination with two-determinant pivoting of $A$ also produces matrices $\hat{A}^{(t)}(t=1, \ldots, r)$ with $\hat{A}^{(t)}[t, \ldots, m \mid t, \ldots, n]$ of the same strict sign of $A$ and the associated permutations also occur in steps $k_{i} \leq r-t$.

Proof. Let us prove by induction on $r$ that, for sufficiently high finite precision arithmetic, the elimination procedure of an $m \times n$ SSR matrix $A$ leads to a sequence of computed matrices $\hat{A}^{(t)}$ such that $\hat{A}^{(t)}[t, \ldots, m \mid t, \ldots, n]$ are SSR and $\hat{A}^{(t)}$ tend to $A^{(t)}$ when the roundoff unit $u$ tends to zero.

The result is trivial for $r=1$. Let us assume that it holds for $r-1$ and let us prove it for $r$. Since $\hat{A}^{(1)}=A$ and $A$ is SSR, one step of Neville elimination with two-determinant pivoting of $A$ can be applied, producing $\tilde{A}^{(1)}$ and $\hat{A}^{(2)}$. The matrix $A^{(2)}[2, \ldots, m \mid 2, \ldots, n]$ is SSR by Proposition 2.1 , the first row of $A^{(2)}$ coincides with the first row of $\tilde{A}^{(1)}$ and in places $(2,1), \ldots,(m, 1) A^{(2)}$ has zeros. By formula (2.2), $\hat{A}^{(2)}$ also has zeros in entries $(2,1), \ldots,(m, 1)$, and its first row coincides with that of $A^{(2)}$. The elements $a_{i j}^{(2)}(2 \leq i \leq m, 2 \leq j \leq n)$ can be expressed as a quotient of determinants of $\tilde{A}^{(1)}$ by the Schur complement formula (2.4). So, since $\tilde{A}^{(1)}$ is SSR, by the continuity of the determinants, we have that $\hat{A}^{(2)}[2, \ldots, m \mid 2, \ldots, n]$ tends to $A^{(2)}[2, \ldots, m \mid 2, \ldots, n]$ when $u$ tends to zero. In conclusion, $\hat{A}^{(2)}$ tends to $A^{(2)}$ when $u$ tends to zero. Moreover, since any determinant of $A^{(2)}[2, \ldots, m \mid 2, \ldots, n]$ is a Schur complement of a minor of the SSR matrix $\tilde{A}^{(1)}$, again by (2.4) and the continuity of the determinant, we can deduce that $\hat{A}^{(2)}[2, \ldots, m \mid 2, \ldots, n]$ is SSR for $u$ sufficiently small. Then $\hat{A}^{(2)}[2, \ldots, m \mid 2, \ldots, n]$ satisfies the induction hypothesis and so $\hat{A}^{(t)}[t, \ldots, m \mid t, \ldots, n]$ is SSR for all $t \geq 3$ for $u$ sufficiently small, and the matrices $\hat{A}^{(t)}[2, \ldots, m \mid 2, \ldots, n]$ tend to $A^{(t)}[2, \ldots, m \mid 2, \ldots, n]$ when $u$ tends to zero. Therefore $\hat{A}^{(t)}$ also tends to $A^{(t)}$ when $u$ tends to zero, and the induction holds.

Since we have seen that for each $t=1, \ldots, r$ the matrix $\hat{A}^{(t)}[t, \ldots, m \mid t, \ldots, n]$ tends to $A^{(t)}[t, \ldots, m \mid t, \ldots, n]$, which is SSR with the sign of $A$ by Proposition 2.1, we conclude that $\hat{A}^{(t)}[t, \ldots, m \mid t, \ldots, n]$ has the same strict sign as $A$.

Finally, since $A^{(t)}[t, \ldots, m \mid t, \ldots n]$ is $\operatorname{SSR}$ for $t=1, \ldots, r$, then

$$
\operatorname{det}\left(A^{(t)}[t, t+1]\right) \neq 0
$$


for $t=1, \ldots, r-1$. Taking into account that determinants are continuous functions, since $\hat{A}^{(t)}$ tends to $A^{(t)}$ when the unit roundoff tends to zero, for a sufficiently high precision $\operatorname{det}\left(\hat{A}^{(t)}[t, t+1]\right)$ has the same strict $\operatorname{sign}$ of $\operatorname{det}\left(A^{(t)}[t, t+1]\right)$ for $t=1, \ldots, r-1$. So, the last statement of the proposition follows.

Now, we compare the behavior of the test for strict sign regularity introduced in Section 4 with that of [7]. For this purpose, given an SSR matrix $A$, we apply the test to $A$ checking if it is SSR. We use MATLAB with floating point arithmetic of finite precision for numerical experiments. We also carry out an analogous experimentation with the test for strict sign regularity appearing in [7. Roughly speaking, the test of [7] uses Neville elimination without row and column exchanges. As Theorem 6.1 and Example 6.2 have shown, the growth factor of Neville elimination with two-determinant pivoting clearly has a better behavior than Neville elimination without permutations. Now, we shall illustrate how these tests work for checking the strict sign regularity of a matrix in finite precision arithmetic. We have chosen the matrix $A$ of the following example because it is very easy to justify that it is SSR and its construction is very simple.

We consider a nonsingular $n \times n$ matrix $B$ given by

$$
B=F_{1} \ldots F_{n-1} G_{n-1} \ldots G_{1}
$$

with $F_{i}, G_{i}$ of the form

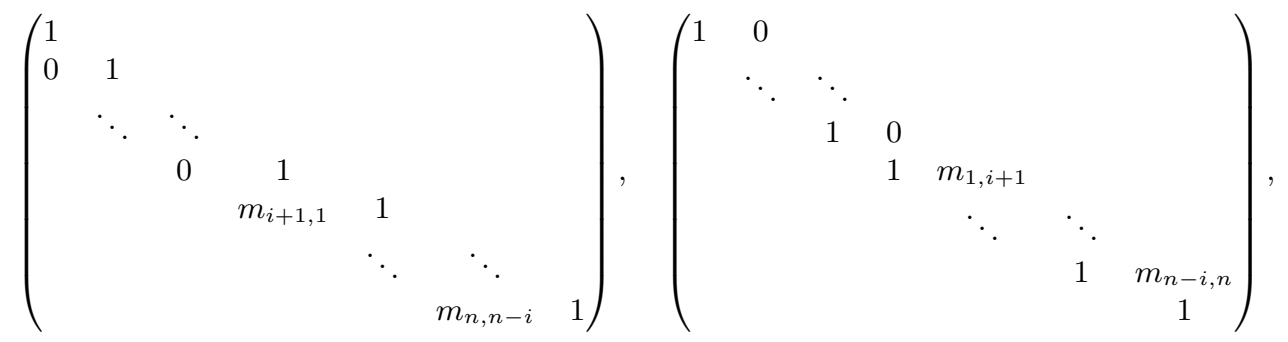

respectively, for $i=1, \ldots, n-1$.

If $m_{h j}, m_{j h}>0$ for all $h=i+1, \ldots, n$ and $j=1, \ldots, n-i$, then by Theorem 4.3 of $[8, B$ is totally positive. Let us consider the matrix $C$ with the form of $B$ obtained by taking the simplest choice $m_{h j}=m_{j h}=1$ for all $h=i+1, \ldots, n$ and $j=1, \ldots, n-i$.

If $A:=P C$, where $P$ is the backward identity matrix, then $A$ is SSR. For $n=10$, we use Matlab with floating point arithmetic of a minimun number of 4 digits in order to get $\hat{A}^{(1)}=A$. Then, the test associated to Neville elimination with twodeterminant pivoting already gives an affirmative answer (' $A$ is SSR') with 4 digits of precision. However, the test associated with [7] needs at least of 6 digits to obtain that $A$ is SSR. For $n=15$, we have to consider 7 digits in order to obtain that $\hat{A}^{(1)}=A$. Then, the test associated to Neville elimination with two-determinant pivoting gives an affirmative answer with 7 digits of precision. However, the test associated to Neville elimination without permutations needs at least 11 digits to obtain that $A$ is SSR. 


\section{REFERENCES}

[1] T. Ando, Totally positive matrices, Linear Algebra Appl. 90 (1987), 165-219. MR884118 (88b:15023)

[2] L.D. Brown, I.M. Johnstone and K.B. MacGibbon, Variation diminishing transformations: a direct approach to total positivity and its statistical applications, Journal of the American Statistical Association 76 (1981), 824-832. MR650893 (83f:62028)

[3] J.M. Carnicer and J.M. Peña, On transforming a Tchebycheff system into a strictly totally positive system, J. Approx. Theory 81 (1995), 274-295. MR1327172 (96a:41036)

[4] V. Cortés and J.M. Peña, Sign regular matrices and Neville elimination, Linear Algebra Appl. 421 (2007), 53-62. MR2290685

[5] S.M. Fallat and P. van den Driessche, On matrices with all minors negative, Electronic Journal of Linear Algebra 7 (2000), 92-99. MR.1779434 (2001g:15026)

[6] M. Gasca and J.M. Peña, Total positivity and Neville elimination, Linear Algebra Appl. 165 (1992), 25-44. MR1149743 (93d:15031)

[7] M. Gasca and J.M. Peña, A Test for Strict Sign-Regularity, Linear Algebra Appl. 197-198 (1994), 133-142. MR1275612 (95a:15022)

[8] M. Gasca and J.M. Peña, On factorizations of totally positive matrices, Total Positivity and Its Applications (1996), 109-130. MR1421600 (97h:15008)

[9] S. Karlin, Total Positivity, Stanford University Press, 1968. MR0230102 (37:5667)

[10] P. Koev and F. Dopico, Accurate eigenvalues of certain sign regular matrices, Linear Algebra Appl. 424 (2007), 435-447. MR2329485

[11] G. Mühlbach and M. Gasca, A test for strict total positivity via Neville elimination, Current trends in matrix theory (1987), 225-232. MR898910 (88h:15040)

[12] J.M. Peña (Ed.), Shape Preserving Representations in Computer-Aided Geometric Design, Nova Science Publishers, 1999.

[13] J.M. Peña, On nonsingular sign regular matrices, Linear Algebra Appl. 359 (2003), 91-100. MR.1948436 (2003k:15031)

[14] J.M. Peña, Characterizations and stable tests for the Routh-Hurwitz conditions and total positivity, Linear Algebra Appl. 393 (2004), 319-332. MR2098621 (2005f:93084)

[15] J.M. Peña, A stable test to check if a matrix is a nonsingular M-matrix, Math. Comp. 73 (2004), 1385-1392. MR2047092 (2004m:15031)

[16] I.J. Schoenberg, Uber variationsverminderende lineare transformationen, Math. Z. 32 (1930), 321-328. MR1545169

Departamento de Matemática Aplicada, Universidad de Zaragoza, 50009, Zaragoza, SPAIN

E-mail address: vcortes@unizar.es

Departamento de Matemática Aplicada, Universidad de Zaragoza, 50009, Zaragoza, SPAIN

E-mail address: jmpena@posta.unizar.es 